\title{
ON THE COORDINATE RING OF A PROJECTIVE POISSON SCHEME
}

\author{
D. KALEDIN
}

\section{Introduction}

Let $Y$ be a smooth symplectic algebraic variety over a field $k$ of characteristic 0 , or, more generally, a Poisson scheme over $k$. Assume that a reductive group $G$ acts on $Y$ preserving the Poisson structure. Moreover, assume that the $G$ action is Hamiltonian - namely, that there exists a Lie algebra homomorphism $\mu: \mathfrak{g} \rightarrow H^{0}\left(Y, \mathcal{O}_{Y}\right)$ from the Lie algebra $\mathfrak{g}$ of the group $G$ to the Lie algebra of global functions on $X$ such that any $\xi \in \mathfrak{g}$ acts on $\mathcal{O}_{Y}$ by taking Poisson bracket with the function $\mu(\xi)$. Then there exists a well-known procedure of taking quotient with respect to the $G$-action, appropriate for the category of Poisson schemes. The procedure is called Hamiltonian reduction. It consists of two steps. Firstly, one considers the map $\mu$ as a so-called moment map $\mu: Y \rightarrow \mathfrak{g}^{*}$ from $Y$ into the affine space $\mathfrak{g}^{*}$ carrying the coadjoint representation of $G$. Secondly, one replaces $Y$ with the zero fiber $Y_{0}=\mu^{-1}(0) \subset Y$ of the moment map and takes the usual, suitably interpreted quotient $Y_{0} / / G$ with respect to the $G$-action (usually the quotient is taken in the sense of GIT). If the group $G$ is abelian, one can also do the steps in the opposite order: firstly, one takes the quotient $Y / / G$, then takes the zero fiber $X=(Y / / G)_{0}$. In any case, the resulting scheme $X=Y_{0} / / G$ is automatically Poisson; moreover, if $Y$ is symplectic and $X$ is smooth, then $X$ is symplectic as well.

The goal of this note is to show how to invert the Hamiltonian reduction procedure in the particular case when the group $G$ is the multiplicative group $\mathbb{G}_{m}$. We start with a Poisson scheme $X$ and a line bundle $L$ on the scheme $X$. We construct, under certain assumptions, a Poisson deformation $\mathfrak{X} / S$ of the scheme $X$ over the formal disc $S=\operatorname{Spec} k[[t]]$ and a line bundle $\mathcal{L}$ on $\mathfrak{X}$ extending $L$. Then we prove that the total space $Y$ of the corresponding $\mathbb{G}_{m}$-principal bundle on $\mathfrak{X}$ is a Poisson scheme, and that the natural $G$-action on $Y$ is Hamiltonian, with the projection $Y \rightarrow \mathfrak{X} \rightarrow S$ giving the moment map. Thus $X$ is the reduction of the Poisson scheme $Y$ with respect to a Hamiltonian $\mathbb{G}_{m}$-action. Moreover, we show that under additional assumptions such a deformation $\mathfrak{X} / S$ is essentially unique.

It seems natural to approach this problem by means of the general Poisson deformation theory, as described in, for instance, the paper [GK]. However,

Received by the editors April 28, 2004.

Partially supported by CRDF grant RM1-2354-MO-02. 
it turns out that there exists a simpler proof. In fact, our proof is almost elementary; the only slightly non-trivial technique that we use is the notion of a stack of groupoids. We generally follow the lines of the paper [KV], where a similar (but harder) result was proved for deformations of smooth symplectic manifolds.

Thus, the question of inverting Hamiltonian reduction with respect to $\mathbb{G}_{m}$ admits an answer, which is at once simple and more or less complete. Perhaps the main question the reader would ask at this point is: why should he or she care? - after all, the question itself is dangerously close to pure sophistry. The author would like to quote the usual meek excuse of "the result being interesting in its own right", and add, as an explanation, that he arrived at the problem while studying Poisson schemes that are projective. Intuitively, the projective coordinate ring of such a scheme ought to carry a Poisson structure, but it does not (and this problem was noted already by M. Kontsevich in [K]). Our theorem explains why not: one has to add an additional deformation. This might be useful in the study of quantizations of such schemes.

We were not able to extend our result to arbitrary reductive groups, and it might be that such a generalization simply does not exist. However, aside from certain cohomology vanishing, we impose absolutely no restrictions on $X$ : it may be singular, non-normal, reducible, non-reduced.

\section{Statements and definitions}

Fix a base field $k$ of characteristic char $k=0$. By a Poisson algebra A over $k$ we will understand a unital commutative algebra $A$ over $k$ equipped with a skew-linear operation $\{-,-\}: \Lambda_{k}^{2}(A) \rightarrow A$ such that

$$
\{a, b c\}=\{a, b\} c+\{a, c\} b, \quad 0=\{a,\{b, c\}\}+\{b,\{c, a\}\}+\{c,\{a, b\}\},
$$

for all $a, b, c \in A$. The operation $\{-,-\}$ is called the Poisson bracket. By the Poisson center of a Poisson algebra $A$ we will understand the subspace of elements $a \in A$ such that $\{a, A\}=0$. By a Poisson module $M$ over the algebra $A$ we will understand an $A$-module $M$ equipped with a Poisson bracket operation $\{-,-\}: A \otimes_{k} M \rightarrow M$ such that

$$
\begin{aligned}
\{a,\{b, m\}\} & =\{b,\{a, m\}\}+\{\{a, b\}, m\}, \\
\{a, b m\} & =\{a, b\} m+b\{a, m\}, \\
\{a b, m\} & =a\{b, m\}+b\{a, m\},
\end{aligned}
$$

for all $a, b \in A, m \in M$. By a Poisson scheme $X$ we will understand a scheme $X$ equipped with a Poisson bracket in its structure sheaf. By a Poisson sheaf over a Poisson scheme $X$ we will understand a coherent sheaf $\mathcal{E}$ of $\mathcal{O}_{X}$-modules equipped with a Poisson bracket. Note that the spectrum Spec $A$ of a Poisson algebra $A$ is an affine Poisson scheme, and the localization $\widetilde{M}$ of a Poisson $A$-module $M$ is a Poisson sheaf on Spec $A$. By a Poisson line bundle we will understand a Poisson sheaf which is flat (equivalently, locally trivial in Zariski 
topology) and has rank 1 . Given a line bundle $\mathcal{L}$ on a scheme $X$, we will denote by

$$
\operatorname{Tot}(\mathcal{L})=\mathcal{S} \text { pec } \bigoplus_{p \in \mathbb{Z}} \mathcal{L}^{p}
$$

the total space of $\mathcal{L}$ without a zero section. Then if $\mathcal{L}$ and $X$ are Poisson, the scheme $\operatorname{Tot}(\mathcal{L})$ is also Poisson. If a Poisson scheme $X$ is equipped with a map $f: X \rightarrow S$ to another scheme $S$, we will say that $X$ is Poisson over $S$ whenever the subsheaf $f^{-1} \mathcal{O}_{s} \subset \mathcal{O}_{X}$ lies in the Poisson center of the algebra sheaf $\mathcal{O}_{X}$.

Remark 1.1. Explictly, the Poisson bracket on $\operatorname{Tot}(\mathcal{L})$ for a Poisson line bundle $\mathcal{L}$ is defined as follows. Local functions on $\operatorname{Tot}(\mathcal{L})$ are linear combinations of local sections $s_{n} \in H^{0}\left(U, \mathcal{L}^{\otimes n}\right), U \subset \mathfrak{X}, n \in \mathbb{Z}$. It suffices to define $\left\{s_{n}, s_{m}\right\}$, and it suffices to do it locally on $\mathfrak{X}$. Choose a trivialization $s \in H^{0}(U, \mathcal{L})$ over some Zariski open $U \subset \mathfrak{X}$; then we have $s_{n}=f s^{n}$ and $s_{m}=g s^{m}$ for some functions $f, g \in H^{0}\left(U, \mathcal{O}_{U}\right)$. By definition, we must have

$$
\begin{aligned}
\left\{s_{n}, s_{m}\right\} & =\left\{f s^{n}, g s^{m}\right\} \\
& =\{f, g\} s^{n+m}+(m g\{f, s\}-n f\{g, s\}) s^{n+m-1}+n m f g\{s, s\} s^{n+m-2} \\
& =\{f, g\} s^{n+m}+(m g\{f, s\}-n f\{g, s\}) s^{n+m-1},
\end{aligned}
$$

where $\{f, s\},\{g, s\} \in H^{0}(U, \mathcal{L})$ are defined by the Poisson structure on the line bundle $\mathcal{L}$. It elementary to check that this does not depend on the choice of the trivialization $s$.

Let $X$ be a Poisson scheme. Any function $f$ on $X$ defines a vector field $H_{f}$ on $X$ by setting

$$
H_{f}(g)=\{f, g\}
$$

for any (local) function $g \in \mathcal{O}_{X}$. The vector field $H_{f}$ is called the Hamiltonian vector field of the function $f$. It vanishes if and only if the function $f$ lies in the Poisson center of $\mathcal{O}_{X}$. Moreover, for any Poisson sheaf $\mathcal{E}$ on $X$ (1.1) defines a differential operator $H_{f}: \mathcal{E} \rightarrow \mathcal{E}$ of order 1 . If $f$ is central, so that $H_{f}=0$ on $\mathcal{O}_{X}$, then $H_{f}: \mathcal{E} \rightarrow \mathcal{E}$ is a $\mathcal{O}_{X}$-linear map.

We will say that a Poisson scheme $X$ is non-degenerate at a smooth closed point $x \in X$ if the whole tangent space $T_{x}$ is spanned by Hamiltonian vector fields $H_{f}$ corresponding to local functions $f \in \mathcal{O}_{X, x}$ on $X$ near $X$. If $X$ is smooth, we will say that it is symplectic if it is non-degenerate at all point. It is well-known that this is equivalent to saying that $X$ carries a non-degenerate closed 2-form $\Omega \in H^{0}\left(X, \Omega_{X}^{2}\right)$ (this is the standard definition nowadays, but historically, the Poisson definition came first).

Remark 1.2. In the case when the Poisson scheme $X$ is non-degenerate, a Poisson module is tautologically the same as a $\mathcal{D}$-module - that is, a sheaf of modules over the algebra $\mathcal{D}_{X}$ of differential operators on $X$. In particular, the category of Poisson modules does not depend on the symplectic structure. In general, the forgetful functor from Poisson modules to coherent sheaves on $X$ admits a left-adjoint, $\mathcal{E} \mapsto U_{X} \otimes \mathcal{E}$, where $U_{X}$ is a certain algebra sheaf on $X$, a 
sort of an universal enveloping algebra for the Poisson structure. Explicitly, the algebra $U_{X}$ is generated by $\mathcal{O}_{X}$ and $\Omega_{X}^{1}$, subject to relations $[a,(d b)]=\{a, b\}$ and $[(d a),(d b)]=d\{a, b\}$. Just as the algebra $\mathcal{D}_{X}$, the algebra $U_{X}$ has an increasing filtration by order; the associated graded algebra $\operatorname{gr} U_{X}$ is isomorphic to the symmetric algebra $S^{\bullet}\left(\Omega^{1}(X)\right)$. If the Poisson structure is non-degenerate, we have $\Omega^{1}(X) \cong \mathcal{T}(X)$ and $U_{X} \cong \mathcal{D}_{X}$. In the general case the algebra $U_{X}$ is probably useless; if $X$ is smooth, it might be convenient for some applications. However, we will not need it, so we skip all the details.

Denote by $S=$ Spec $k[[t]]$ the formal disc over $k$. For any Poisson scheme $X$ equipped with a map $f: X \rightarrow S$, the pullback $f^{*} t$ of the parameter $t$ gives a global function on $X$, and we have the Hamiltonian vector field $H_{t}$. By our convention, $X$ is a Poisson scheme over $S$ if and only if $H_{t}=0$. Another important situation is when $H_{t}$ can be integrated to give a $\mathbb{G}_{m}$-action on $X$. In this case, we say that the $\mathbb{G}_{m}$-action is Hamiltonian, and that $f: X \rightarrow S$ is the moment map for the $\mathbb{G}_{m}$-action. Being a Hamiltonian vector field, $H_{t}$ is in particular a derivation with respect to the Poisson bracket; therefore a Hamiltonian $\mathbb{G}_{m}$-action preserves the Poisson structure.

We leave it to the reader to check that all of the above make sense verbatim if one allows $X$ to be a formal scheme.

Definition 1.3. A formal moment system is the pair $\langle\mathfrak{X} / S, \mathcal{L}\rangle$ of a flat Poisson formal scheme $\mathfrak{X} / S$ and a Poisson line bundle $\mathcal{L}$ on $\mathfrak{X}$ such that

(i) the Hamiltonian vector field $H_{t}$ vanishes on $\mathcal{O}_{X}(X$ is Poisson over $S)$, and

(ii) the $\mathcal{O}_{X}$-linear map $H_{t}: \mathcal{L} \rightarrow \mathcal{L}$ is equal to the identity map.

Note that since $\mathfrak{X}$ is Poisson over $S$, the fiber $\mathfrak{X}_{o}=\mathfrak{X} \times_{S}$ o of the scheme $\mathfrak{X}$ over the closed point $o \in X$ is also a Poisson scheme, and the line bundle $\mathcal{L}$ gives by restriction a line bundle $L$ on $\mathfrak{X}_{o}$. We will say that the moment system $\langle\mathfrak{X} / S, \mathcal{L}\rangle$ extends the pair $\left\langle\mathfrak{X}_{o}, L\right\rangle$. Our main result is the following.

Theorem 1.4. Assume given a Poisson scheme $X$ and a line bundle $\mathcal{L}$ on $X$.

(i) Assume that $H^{2}\left(X, \mathcal{O}_{X}\right)=0$. Then there exists a formal moment system $\langle\mathfrak{X} / S, \mathcal{L}\rangle$ extending the pair $\langle X, L\rangle$.

(ii) Assume in addition that $H^{1}\left(X, \mathcal{O}_{X}\right)=0$. Then such a moment system is unique up to an isomorphism.

In applications, one often has one additional piece of structure on a Poisson scheme $X$ - namely, a vector field $\xi$ which is conformal of weight $\lambda \in k$ in the sense that

$$
\xi(\{f, g\})=\{\xi(f), g\}+\{f, \xi(g)\}+\lambda\{f, g\} .
$$

Theorem 1.4 is compatible with such a vector field in the following sense.

Proposition 1.5. In the assumptions of Theorem 1.4 (ii), assume in addition that $X$ is equipped with a conformal vector field $\xi$ of some weight $\lambda$. Then $\xi$ extends to a vector field on $\mathfrak{X}$ such that $\xi()=\lambda$, and $\mathcal{L}$ admits an action of $\xi$ compatible with the Poisson module structure. 
To state our second result, say that a scheme $X$ is algebraically convex (by analogy with holomorphically convex spaces) if the natural morphism

$$
X \rightarrow \operatorname{Spec} H^{0}\left(X, \mathcal{O}_{X}\right)
$$

is a projective map.

Theorem 1.6. In the assumptions of Theorem 1.4, assume that in addition to $H^{1}\left(X, \mathcal{O}_{X}\right)=H^{2}\left(X, \mathcal{O}_{X}\right)=0$, the scheme $X$ is algebraically convex. Then the formal scheme $\mathfrak{X}$ can be extended to an actual algebraically convex scheme which is flat over $S$. The algebra $H^{0}\left(\mathfrak{X}, \mathcal{O}_{\mathfrak{X}}\right)$ of global functions on $\mathfrak{X}$ is flat over $k[[t]]$ and $t$-adically complete, the natural map $H^{0}\left(\mathfrak{X}, \mathcal{O}_{X}\right) / t \rightarrow H^{0}\left(X, \mathcal{O}_{X}\right)$ is an isomorphism, the natural $\mathbb{G}_{m}$-action on the total space $\operatorname{Tot}(\mathcal{L})$ is Hamiltonian, and $\operatorname{Tot}(\mathcal{L}) \rightarrow \mathfrak{X} \rightarrow S$ gives a moment map. If $X$ is a regular scheme, then both $\mathfrak{X}$ and $\operatorname{Tot}(\mathcal{L})$ are regular schemes, while $H^{0}\left(\mathfrak{X}, \mathcal{O}_{\mathfrak{X}}\right)$ is a normal k-algebra. If $X$ is symplectic, then $\operatorname{Tot}(\mathcal{L})$ is symplectic.

1.1. The symplectic case. Assume that the Poisson scheme $X$ is smooth and symplectic, so that the Poisson structure is defined by a symplectic form $\Omega_{X} \in H^{0}\left(X, \Omega_{X}^{2}\right)$. For any deformation $\left\langle\mathfrak{X}, \Omega_{\mathfrak{X}}\right\rangle$ of the pair $\left\langle X, \Omega_{X}\right\rangle$ over a local Artin base $S=\operatorname{Spec} \mathcal{O}_{S}$, the Gauss-Manin connection provides a trivialization $H_{D R}^{\cdot}(\mathfrak{X} / S) \cong H_{D R}^{\cdot}(X) \otimes \mathcal{O}_{S}$ of the relative de Rham cohomology module $H_{D R}^{\bullet}(\mathfrak{X} / S)$. The cohomology class $\left[\Omega_{\mathfrak{X}}\right] \in H_{D R}^{2}(\mathfrak{X} / S)$ need not be constant with respect to this trivialization; on the contrary, one defines the period map $P: S \rightarrow H_{D R}^{2}(X)$ as the graph of $\left[\Omega_{\mathfrak{X}}\right] \in H_{D R}^{2}(\mathfrak{X} / S) \cong H_{D R}^{2}(X) \otimes \mathcal{O}_{S}$, and it has been shown in $[\mathrm{KV}]$ that the period map completely defines the deformation (in particular, $P$ is constant if and only if $\mathfrak{X} \cong X \times S$ and $\Omega_{\mathfrak{X}}=\Omega_{X}$ ). The moment systems are described in this language in the following way.

Lemma 1.7. Assume given a line bundle $L$ on a symplectic manifold $\langle X, \Omega\rangle$, and a formal moment system $\langle\mathfrak{X} / S, \mathcal{L}\rangle, S=\operatorname{Spec} k[[t]]$. Then the Poisson scheme $\mathfrak{X} / S$ is non-degenerate, and the corresponding relative symplectic form $\Omega_{\mathfrak{X}}$ on $\mathfrak{X} / S$ satisfies

$$
\left[\Omega_{\mathfrak{X}}\right]=\left[\Omega_{X}\right]+[L] t \in H_{D R}^{2}(\mathfrak{X} / S) \cong H_{D R}^{2}(X) \otimes \mathcal{O}_{S}=H_{D R}^{2}(X)[[t]],
$$

where $[L] \in H_{D R}^{2}(X)$ is the first Chern class of the line bundle $L$.

Proof. By definition, for any smooth family $\mathfrak{X} / S$ and a closed relative differential form $\alpha \in H^{0}\left(\mathfrak{X}, \Omega_{\mathfrak{X} / S}^{\bullet}\right)$, one can compute the Gauss-Manin connection $\nabla[\alpha] \in$ $H_{D R}^{l}(\mathfrak{X} / S) \otimes \Omega_{S}^{1}$ by the following procedure: one lifts the relative form $\alpha$ to an absolute form $\widetilde{\alpha} \in H^{0}\left(\mathfrak{X}, \Omega_{\mathfrak{X}}^{\bullet}\right)$, one notices that $d \widetilde{\alpha}=d \alpha=0 \bmod \Omega_{S}^{\geq 1}$, and, independently of the choice of a lifting, one has $[d \widetilde{\alpha}]=\nabla[\alpha] \bmod \Omega_{\bar{S}}^{\geq 2}$, where both sides are hypercohomology classes with coefficients in

$$
\Omega_{\mathfrak{X} / S}^{\cdot} \otimes \Omega_{S}^{1} \cong\left(\Omega_{\mathfrak{X}}^{\bullet} \cdot \Omega_{S}^{\geq 1}\right) /\left(\Omega_{\mathfrak{X}}^{\bullet} \cdot \Omega_{\bar{S}}^{\geq 2}\right) \text {. }
$$

Denote by $Y=\operatorname{Tot}(\mathcal{L})$ the total space of the line bundle $\mathcal{L}$ without the zero section, and let $\pi: Y \rightarrow \mathfrak{X}$ be the natural projection. To compute $H_{D R}^{\bullet}(\mathfrak{X} / S)$, 
one can use the equivariant de Rham complex $\Omega_{\mathbb{G}_{m}}(Y)$ (see, e.g., [BGV]). Recall that we have

$$
\Omega_{\mathbb{G}_{m}}(Y)=\Omega^{\bullet}(Y) \otimes k[u],
$$

where $u$ is an additional generator of degree 2 ; the differential $d^{\mathbb{G}_{m}}$ in the equivariant de Rham complex is given by $d^{\mathbb{G}_{m}}(\alpha)=d \alpha+u i_{\xi}(\alpha)$, where $i_{\xi}$ is the contraction with the differential $\xi \in H^{0}\left(Y, \mathcal{T}_{Y}\right)$ of the $\mathbb{G}_{m}$-action on $Y$. By definition, the natural map $\pi^{*} \Omega_{\mathfrak{X} / S} \rightarrow \Omega_{Y}^{\bullet}$ extends to a map

$$
\pi^{*} \Omega_{\mathfrak{X} / S} \rightarrow \Omega_{\mathbb{G}_{m}}^{\cdot}(Y)
$$

which induces an isomorphism on hypercohomology groups.

Now, the relative symplectic form $\pi^{*} \Omega_{\mathfrak{X}} \in H^{0}\left(Y, \Omega_{\mathbb{G}_{m}}^{2}(Y)\right)$ by definition comes from the absolute symplectic form $\Omega_{Y} \in H^{0}\left(Y, \Omega_{Y}^{2}\right)$. The form $\Omega_{Y}$ is not closed in the equivariant de Rham complex: while $d \Omega_{Y}=0, i_{\xi} \Omega_{Y}$ is non-trivial. In fact, since the projection $Y \rightarrow S$ is the moment map for the $\mathbb{G}_{m}$-action on $Y$, we have $i_{\xi} \Omega_{Y}=d t$. We conclude that

$$
\nabla\left[\Omega_{\mathfrak{X}}\right]=\nabla\left[\pi^{*} \Omega_{\mathfrak{X}}\right]=\left[d^{\mathbb{G}_{m}} \Omega_{Y}\right]=\left[u i_{\xi} \Omega_{Y}\right]=[u] d t .
$$

It remains to notice that by definition, we have $\pi^{*}[L]=u \in H^{2}\left(Y, \Omega_{\mathbb{G}_{m}}^{\cdot}(Y)\right) \cong$ $H_{D R}^{2}(\mathfrak{X} / S)$. Therefore $\nabla\left[\Omega_{\mathfrak{X}}\right]=[L] d t$, which, since $\left[\Omega_{\mathfrak{X}}\right]=\left[\Omega_{X}\right] \bmod t$, implies (1.3).

To understand better the relation between symplectic deformations and the moment systems, assume that $X$ is a symplectic manifold with

$$
H^{1}\left(X, \mathcal{O}_{X}\right)=H^{2}\left(X, \mathcal{O}_{X}\right)=0
$$

Then the main theorem of $[\mathrm{KV}]$ claims that $X$ admits a universal symplectic formal deformation $\mathfrak{X} / C$ over a smooth base $C$. The corresponding period map $P: C \rightarrow H_{D R}^{2}(X)$ identifies $C$ with the formal neighborhood of the cohomology class $\left[\Omega_{X}\right] \in H_{D R}^{2}(X)$ in the affine space $H_{D R}^{2}(X)$. Then Lemma 1.7 shows that a moment system associated with a line bundle $L$ is parametrized by the line $\left[\Omega_{X}\right]+t[L] \in C$ in the base $C$ of the universal deformation $\mathfrak{X} / C$.

The methods of $[\mathrm{KV}]$ work only in the symplectic situation; moreover, they do not give a Poisson line bundle $\mathcal{L}$. On the other hand, the approach of $[\mathrm{KV}]$ requires weaker cohomological assumptions on $X$ and, more importantly, it provides a multi-parameter deformation $\mathfrak{X} / C$ which is universal in appropriate sense. However, for general Poisson schemes - for instance, for a Poisson scheme with zero Poisson bracket - the deformation theory behaves in an unpredictable way, and there is no reason to expect that a universal Poisson deformation even exists, let alone is smooth. So, in general, Theorem 1.4 is not far from an optimal possible result on Poisson deformations.

\section{Proofs}

We will now prove Theorem 1.4, Proposition 1.5 and Theorem 1.6. Our proof proceeds by induction. To set up the induction, for any integer $n \geq 1$ we denote 
by $S_{n}=\operatorname{Spec} k[[t]] / t^{n+1}$ the $n$-th infinitesemal neighborhood of the special point $o \in S$. By definition, we have canonical embeddings

$$
S_{1} \subset \cdots \subset S_{n} \subset \ldots
$$

Definition 2.1. An order-n moment system is a pair $\left\langle X_{n} / S_{n}, \mathcal{L}\right\rangle$ of a flat Poisson scheme $X_{n} / S_{n}$ and a line bundle $\mathcal{L}$ on the subscheme $X_{n-1}=X_{n} \times_{S_{n}} S_{n-1} \subset$ $X_{n}$ equipped with a structure of a Poisson sheaf on $X_{n}$, such that $X_{n} / S_{n}$ and $\mathcal{L}$ satisfy the conditions (i), (ii) of Definition 1.3.

Note that the condition (i) insures that $X_{n-1} \subset X_{n}$ is a Poisson scheme, and $t^{n} \mathcal{L} \subset \mathcal{L}$ is a Poisson subsheaf. Thus, given an order- $n$ moment system $\left\langle X_{n} / S_{n}, \mathcal{L}\right\rangle$, we obtain by restriction a moment system $\left\langle X_{n-1}, \mathcal{L} / t^{n} \mathcal{L}\right\rangle$ of order $n-1$. Analogously, given a formal moment system, we obtain by restriction an order- $n$ moment system for every $n \geq 1$. For $n=0$, we simply obtain a Poisson scheme $X_{0}$. We will say that the given moment system extends the Poisson scheme $X_{0}$.

Remark 2.2. The sheaf $\mathcal{L}$ in Definition 2.1 is not a Poisson module over $X_{n-1}$ (multiplication by $t^{n}$ is trivial on $\mathcal{L}$, but the bracket with $t^{n}$ is not trivial - in fact, up to a constant $H_{t^{n}}$ is equal to the multiplication by $t^{n-1}$ ).

Moment systems of order $n$ form a category, with morphisms (we will only need isomorphisms) defined in the obvious way. For an arbitrary Poisson scheme $X$, the pair $\left\langle X \times S_{n}, \mathcal{O}_{X_{n-1}}\right\rangle$ is obviously an order- $n$ moment system. We will call it trivial. Given an order- $n$ moment system $\left\langle\mathfrak{X}_{n}, \mathcal{L}\right\rangle$, by a trivialization of the sheaf $\mathcal{L}$ we will understand a section $e \in H^{0}\left(X_{n}, \mathcal{L}\right)$ which gives an isomorphism $\mathcal{O}_{X_{n-1}} \rightarrow \mathcal{L}$.

Lemma 2.3. Let $X=\operatorname{Spec} A$ be an affine Poisson scheme. Then for any order$n$ moment system $\left\langle X_{n}, \mathcal{L}\right\rangle$ extending $X$, every trivialization e of the sheaf $\mathcal{L}$ on $X_{n-1}$ extends uniquely to an isomorphism between $\left\langle X_{n}, \mathcal{L}\right\rangle$ and the trivial moment system $\left\langle X \times S_{n}, \mathcal{O}_{X_{n-1}}\right\rangle$.

Proof. Since $X_{n}$ extends an affine scheme $X$, it is itself affine, so that $X_{n}=$ Spec $A_{n}$ for some Poisson algebra $A_{n}$. We have $A_{n} / t \cong A$, and $A_{n-1}=A_{n} / t^{n}$ gives $X_{n-1}=\operatorname{Spec} A_{n-1}$. The sheaf $\mathcal{L}$ corresponds to a $A_{n}$-module which is by assumption identified with $e A_{n-1}$. Denote by

$$
A_{n}^{0} \subset A_{n}
$$

the subalgebra of functions $a \in A_{n}$ such that $\{a, e\}=0$. We claim that the restriction map $A_{n} \rightarrow A_{n} / t \cong A$ induces an isomorphism $A_{n}^{0} \cong A$. Indeed, this is obvious for the trivial moment system. By induction on $n$, we may assume that it is true for $A_{n-1}^{0} \subset A_{n-1}=A_{n} / t^{n}$. Thus it suffices to prove that for every function $a \in A_{n-1}^{0}$, the space $P_{a} \subset A_{n}$ of all functions $b \in A$ such that $b=a \bmod t^{n}$ contains exactly one function $a^{\prime} \in P_{a}$ satisfying $\left\{a^{\prime}, e\right\}=0$. But this is obvious: the space $P_{a}$ is a torsor over $t^{n} A \subset A_{n}$, the commutator $\left\{P_{a}, e\right\} \subset e A_{n-1}$ lies in $t^{n-1} e A_{n-1} \subset e A_{n-1}$, and the commutator map $\{-, e\}$ : $A_{n} \rightarrow e A_{n-1}$ identifies $t^{n} A \subset A_{n}$ with $t^{n-1} e A_{n-1}$. 
To finish the proof, it suffices to notice that $A_{0} \otimes k[t] / t^{n+1} \rightarrow A_{n}$ is a Poisson map, hence an isomorphism between $A_{n}$ and the trivial moment system $A \otimes$ $k[t] / t^{n+1}$.

Let now $X$ be an arbitrary Poisson scheme, and assume given a moment system $\left\langle X_{n}, \mathcal{L}\right\rangle$ of order $n$ which extends $X$. Denote by $\mathrm{L}_{n}$ the groupoid of all line bundles $\mathcal{L}^{\prime}$ on $X_{n}$ equipped with an isomorphism $\mathcal{L}^{\prime} / t^{n} \cong \mathcal{L}$. Denote by $\mathrm{M}_{n}$ the groupoid of all moment systems $\left\langle X_{n+1}, \mathcal{L}\right\rangle$ of order $(n+1)$ equipped with an isomorphism between their restriction to order $n$ and the fixed moment system $\left\langle X_{n}, \mathcal{L}\right\rangle$. As part of the data, every moment system in $\mathrm{M}_{n}$ includes a line bundle on the restriction $X_{n}$, so that we have a natural forgetful functor $\mathrm{M}_{n} \rightarrow \mathrm{L}_{n}$.

Lemma 2.4. The forgetful functor $\mathrm{M}_{n} \rightarrow \mathrm{L}_{n}$ is an equivalence of categories.

Proof. Both $\mathrm{M}_{n}$ and $\mathrm{L}_{n}$ are stacks of groupoids on $X$ in the Zariski topology, and the forgetful functor is compatible with the stack structure. Therefore it suffices to prove the claim locally on $X$. But for affine schemes $X$, the claim follows immediately from Lemma 2.3.

Proof of Theorem 1.4. Construct the desired formal moment system as an inverse limit of order- $n$ moment systems, and construct the order- $n$ moment systems by induction on $n$. The base of the induction is the given Poisson scheme $X=X_{0}$. To establish the induction step, note that If $H^{2}\left(X, \mathcal{O}_{X}\right)=0$, then every line bundle on $X_{n-1}$ extends to a line bundle on $X_{n}$. Moreover, if $H^{1}\left(X, \mathcal{O}_{X}\right)=0$, then such an extension is unique up to an isomorphism. Now apply Lemma 2.4.

Proof of Proposition 1.5. Consider the dual numbers algebra $k\langle\varepsilon\rangle=k[\varepsilon] / \varepsilon^{2}$, let $X^{\prime}=X \times_{k} k\langle\varepsilon\rangle$, and consider two maps $p_{0}, p_{1}: X^{\prime} \rightarrow X-p_{0}$ is the natural projection, and $p_{1}$ is given locally by $p_{1}^{*}(f)=f+\xi(f) \varepsilon$. We can turn $X^{\prime}$ into a Poisson scheme in two ways: the obvious one, which we denote $X_{0}^{\prime}$ (the map $p_{0}: X^{\prime} \rightarrow X$ is then Poisson), and the obvious one with the Poisson bracket multiplied by $\left(1+\lambda \varepsilon\right.$ ), which we denote $X_{1}^{\prime}$ (the map $p_{1}: X_{1}^{\prime} \rightarrow X$ is Poisson). Let $\mathfrak{X}_{i}^{\prime}, i=0,1$ be the moment system associated by Theorem 1.4 to $\left\langle X_{i}^{\prime}, p_{i}^{*} L\right\rangle$. Then since $H^{1}\left(X, \mathcal{O}_{X}\right)=0$, the line bundle $L$ admits an action of $\xi$, so that $p_{0}^{*} L \cong p_{1}^{*} L$. We now notice that if we modify the moment system $\mathfrak{X}_{0}^{\prime}$ by composing the projection $\mathfrak{X}_{0}^{\prime} \rightarrow S$ with the map $S \rightarrow S, t \mapsto \lambda t$, then it becomes a moment system for the pair $\left\langle X_{1}^{\prime}, p_{0}^{*} L \cong p_{1}^{*} L\right\rangle$. By the unicity statement of Theorem 1.4, the two moment systems become isomorphic, which proves the claim.

Proof of Theorem 1.6. Assume that the Poisson scheme $X$ is algebraically convex, so that we have the projective map $X \rightarrow Y=\operatorname{Spec} A$ with $A=H^{0}\left(X, \mathcal{O}_{X}\right)$. Take the canonical formal moment system $\mathfrak{X}$ extending $X$ and its order- $n$ restrictions $X_{n}$. Let $A_{n}=H^{0}\left(X_{n}, \mathcal{O}_{X_{n}}\right)$. Then since $H^{1}\left(X, \mathcal{O}_{X}\right)=0$, the restriction maps $A_{n} \rightarrow A_{n-1}$ are surjective, and we have short exact sequences

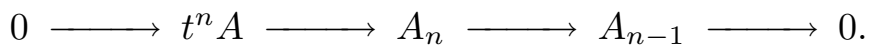


Therefore $A_{\infty}=\lim _{\leftarrow} A_{n}$ is a Noetherian algebra over $k$ which is flat over $k[[t]]$ and complete with respect to the $t$-adic filtration. Since $H^{2}\left(X, \mathcal{O}_{X}\right)=0$, an ample line bundle on $X$ extends to the formal scheme $\mathfrak{X}$. Thus it is a projective formal scheme over $\mathcal{Y}=\operatorname{Spec} A_{\infty}$, and we can apply the Algebraization Theorem [EGA, Théorème 5.4.5]. Moreover, by construction $A_{\infty}=H^{0}\left(\mathfrak{X}, \mathcal{O}_{X}\right)$, so that $\mathfrak{X}$ is indeed algebraically convex.

Consider now $\operatorname{Tot}(\mathcal{L})$. The fact that the natural $\mathbb{G}_{m}$-action is Hamiltonian with the prescribed moment map is obviously precisely equivalent to Definition 1.3 (ii). Assume that $X$ is smooth and symplectic. By construction, we have smooth maps

$$
\operatorname{Tot}(\mathcal{L}) \rightarrow \mathfrak{X} \rightarrow S
$$

whose fibers are, respectively, $\mathbb{G}_{m}$ and $X$. Since $X, S$ and $\mathbb{G}_{m}$ are smooth, so is $\operatorname{Tot}(\mathcal{L})$. It remains to prove that the Poisson structure on $\operatorname{Tot}(\mathcal{L})$ is nondegenerate at every point $z \in \operatorname{Tot}(\mathcal{L})$. The Hamiltonian vector field $H_{t}$ spans the subspace in $T_{z} \operatorname{Tot}(\mathcal{L})$ which is tangent to the $\mathbb{G}_{m}$-orbit. Since $X$ is symplectic, Hamiltonian vector fields $H_{f}$ span the tangent space to $X$ at the image of the point $z$. These vector fields can be lifted to $\operatorname{Tot}(\mathcal{L})$ - indeed, it suffices to lift the defining function. All in all, we see that Hamiltonian vector fields span the relative tangent subspace

$$
T_{z}(\operatorname{Tot}(\mathcal{L}) / S) \subset T_{z} \operatorname{Tot}(\mathcal{L}) .
$$

But this is a subspace of codimension 1 , and it is easy to see that the subspace spanned by Hamiltonian vector fields must be even-dimensional. Thus they must span the whole $T_{z} \operatorname{Tot}(\mathcal{L})$.

\section{Acknowledgments}

I am grateful to R. Bezrukavnikov, D. Huybrechts and A. Kuznetsov for valuable discussions. I am grateful to the referee for a detailed report and important suggestions; in particular, he suggested that Remarks 1.1 and 1.2 would clarify the exposition.

\section{References}

[BGV] N. Berline, E. Getzler, and M. Vergne, Heat kernels and Dirac operators, Grundlehren der Mathematischen Wissenschaften, 298, Springer-Verlag, Berlin, 1992.

[EGA] A. Grothendieck, Éléments de géométrie algébrique, III, Inst. Hautes tudes Sci. Publ. Math., no. 28, 1966.

[GK] V. Ginzburg and D. Kaledin, Poisson deformations of symplectic quotient singularities, Adv. Math. 186 (2004) 1-57.

[KV] D. Kaledin and M. Verbitsky, Period map for non-compact holomorphically symplectic manifolds, Geom. Funct. Anal. 12 (2002) 1265-1295.

[K] M. Kontsevich, Deformation quantization of algebraic varieties, EuroConférence Moshé Flato 2000, Part III (Dijon). Lett. Math. Phys. 56 (2001) 271-294.

Steklov Math Institute, Moscow, USSR

E-mail address: kaledin@mccme.ru 